\title{
ADENOMA VILOSO COM TRANSFORMAÇÃO CARCINOMATOSA DA AMPOLA DE VATER
}

\author{
VILLOUS ADENOMA WITH CARCINOMATOUS DEGENERATION \\ OF THE AMPULLA OF VATER
}

\author{
Guilherme Pinto Bravo Neto, TCBC-RJ ${ }^{1}$ \\ Patrícia Correa de Melo Araújo ${ }^{2}$ \\ Roberta Arinelli Fernandes ${ }^{2}$
}

\section{INTRODUÇÃO}

Os adenomas vilosos do duodeno são lesões raras, compreendendo aproximadamente $1 \%$ dos tumores duodenais e $0,01 \%$ de todas as neoplasias gastroduodenais ${ }^{1}$. A incidência destes tumores, entretanto, pode estar aumentando ou se tornando mais evidente com a utilização em larga escala dos exames endoscópicos ${ }^{2}$. Descrevemos o caso de um paciente com adenoma viloso gigante da segunda porção duodenal, envolvendo a ampola de Vater e tratado por duodenopancreatectomia.

\section{RELATO DO CASO}

Paciente de 47 anos, masculino, branco, procurou atendimento médico devido a icterícia, colúria e desconforto abdominal em hipocôndrio direito, que regrediu espontaneamente após cerca de três dias. Realizou ultra-sonografia que revelou vesícula biliar normodistendida, com lama biliar, e discreta dilatação da via biliar intra-hepática. Colangiorressonância evidenciou falha de enchimento em topografia de colédoco terminal (Figura 1). Endoscopia digestiva alta com duodenoscópio de visão lateral demonstrou volumoso tumor duodenal envolvendo a papila de Vater, sugestivo de tumor viloso. Biópsia endoscópica compatível com adenoma viloso. Levado à cirurgia, evidenciou-se, após abertura do duodeno, extenso tumor vegetante de $2^{\text {a }}$ porção, que ocupava toda a circunferência do órgão, e com extensão de cerca de $6 \mathrm{~cm}$ (Figura 2). Optouse por gastroduodenopancreatectomia cefálica. Houve boa evolução pós-operatória, com alta no 6o PO sem complica- ções. O laudo histopatológico da peça cirúrgica foi de adenoma viloso circunferencial do duodeno, ampola de Vater e colédoco distal, com áreas focais de infiltração da submucosa ( adenocarcinoma).

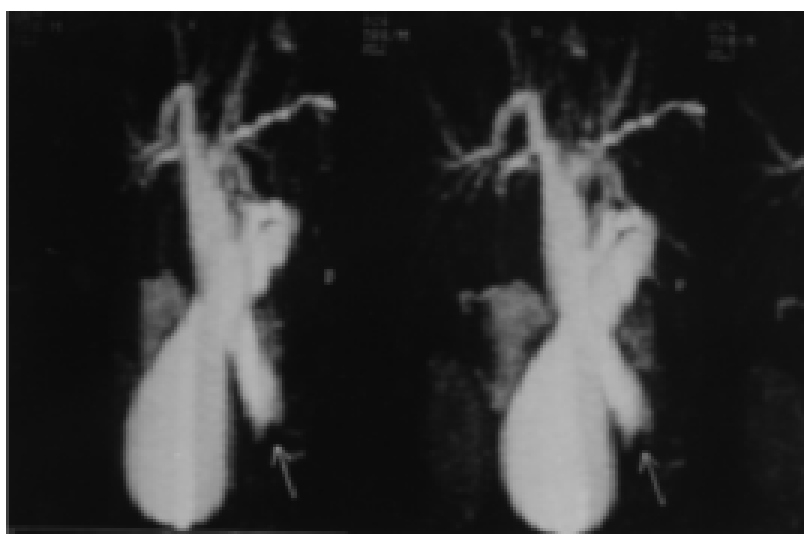

Figura 1 - Colangiorresonância: falha de enchimento em colédoco terminal.

\section{DISCUSSÃO}

Os adenomas vilosos do trato gastrointestinal superior são mais freqüentes no duodeno, e comprometem a região periampular na maioria dos casos. $\mathrm{O}$ diagnóstico clínico destas lesões era raro até o início da década de 1970, quando a colangiopancreatografia retrógrada endoscópica

1. Professor Adjunto do Departamento de Cirurgia da Faculdade de Medicina da UFRJ.

2. Acadêmicas de Medicina da UFRJ.

Recebido em $1 / 8 / 2000$

Aceito para publicação em 26/9/2000

Trabalho realizado no Serviço de Cirurgia Geral do Hospital Universitário Clementino Fraga Filho da Universidade Federal do Rio de Janeiro - UFRJ. 


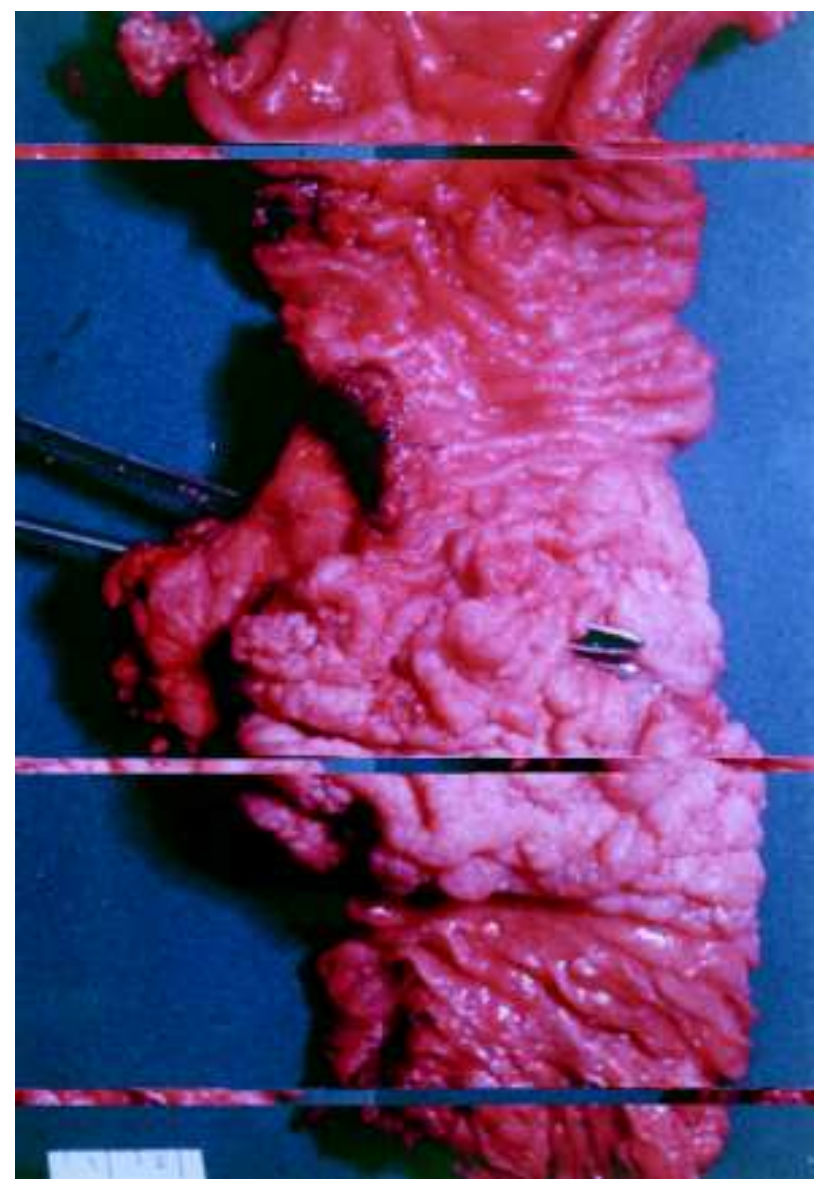

Figura 2 - Peça cirúrgica: volumoso tumor viloso de segunda porção duodenal envolvendo a papila de Vater.

(CPRE) passou a ser largamente utilizada no diagnóstico das doenças biliopancreáticas, aumentando o índice de detecção destes tumores².

Seu pico de incidência ocorre na sétima década de vida e ocorre na mesma proporção em homens e mulheres. Os principais sintomas são dor abdominal e icterícia, esta última presente em mais de $75 \%$ dos pacientes, podendo haver colangite espontânea em alguns casos ${ }^{3}$. Pancreatite aguda também pode ocorrer ${ }^{4}$. Sangue oculto positivo nas fezes e anemia ferropriva são relatados em cerca de $1 / 3$ dos pacientes. Icterícia flutuante, conforme observada em nosso paciente, acompanhada de melena, quadro clássico das neoplasias da ampola de Vater, encontra-se em apenas pequeno número de casos. A duodenoscopia com endoscópios de visão lateral e a CPRE são os métodos diagnósticos de escolha , que permitem ainda a realização de biópsias. Estas, entretanto, devem ser interpretadas com cautela, pois a diferenciação entre adenoma benigno e carcinoma infiltrante costuma ser difícil, com elevado índice de resultados falsos negativos para carcinoma, que podem chegar a $30 \%$ 2,3. Por outro lado, como ocorreu no presente caso, os adenomas vilosos da região periampular são os mais propensos à degeneração maligna quando compara- dos a adenomas de outras localizações no duodeno, com índices de malignização de até $72 \%$ em algumas séries ${ }^{4}$. Ao contrário do que ocorre com os adenomas vilosos do cólon, a malignização não está necessariamente associada ao tamanho da lesão. Além disto, biópsias de congelação não aumentam a acurácia histológica do diagnóstico. A tomografia computadorizada (TC), e mais recentemente a ultrasonografia endoscópica, utilizadas para estadiamento de tumores esofagogastroduodenais, podem também gerar subsídios para o diagnóstico de malignidade, na medida em que fornecem dados sobre infiltração tumoral e presença de metástases ganglionares. Em nosso paciente, o exame microscópico da peça cirúrgica já revelava áreas de infiltração focal da submucosa compatíveis com malignização.

$\mathrm{O}$ aspecto macroscópico dos adenomas vilosos do duodeno e da ampola de Vater é variável, alguns se apresentando como pólipos de base estreita, e outros como tumores sésseis extensos, como o do paciente em questão.

O tratamento de escolha dos adenomas vilosos que comprometem a ampola de Vater é a ressecção. Esta, no entanto, tem sido realizada através de diferentes métodos endoscópicos e cirúrgicos, na dependência de diversos fatores tais como extensão da lesão, aspecto macroscópico e grau de comprometimento ductal biliopancreático. As ressecções endoscópicas, apesar de atrativas por serem menos invasivas, possuem altas taxas de recidiva, de até $26 \%$ em algumas séries e índices de complicações como hemorragia e pancreatite de até $20 \%$, além de serem inadequadas do ponto de vista oncológico, nos casos em que há degeneração maligna ${ }^{2}$. A cirurgia endoscópica pode ser utilizada também como método paliativo em casos de obstrução biliopancreática, através de esfincterotomias e colocação de endopróteses.

A ampulectomia é uma opção cirúrgica defendida por alguns autores, particularmente em pacientes de elevado risco cirúrgico, porém é contra-indicada nos adenomas vilosos com base maior do que $3 \mathrm{~cm}$ ou que infiltrem o ducto biliar ou pancreático por mais de $1,5 \mathrm{~cm}$ de extensão 2,3. Mandatória também é a avaliação inequívoca de benignidade nestes casos, o que nem sempre é possível. Lesões com características histológicas duvidosas e principalmente as lesões mais extensas, como a relatada, devem ser tratadas por duodenopancreatectomia ${ }^{\mathbf{3 , 4}}$. A operação de Whipple tem se mostrado um método seguro, com taxas de mortalidade abaixo de $4 \%$ em vários grandes centros, e vem se tornando o procedimento de escolha para pacientes portadores de diversas doenças benignas e malignas da região periampular ${ }^{4}$. Duodenectomia sem pancreatectomia, também vem sendo indicada em casos selecionados por alguns autores ${ }^{1,5}$.

Tendo em vista a dificuldade em se estabelecer um diagnóstico pré-operatório de malignidade, é nossa opinião de que o procedimento de Whipple representa o método terapêutico de escolha em adenomas vilosos da ampola de Vater, reservando as ressecções locais transduodenais para lesões pequenas, em pacientes com risco cirúrgico elevado. 


\begin{abstract}
Villous adenomas of the duodenum and ampulla of Vater are uncommon, but they have been diagnosed more frequently with the increasing use of upper gastrointestinal endoscopy. Differential diagnosis with villous adenocarcinoma may be difficult. The authors present a case of a 47-year-old man with a giant villous adenoma of the duodenum, with intermittent jaundice, that was treated by pancreatoduodenectomy.
\end{abstract}

Key Words: Villous adenoma; Adenocarcinoma; Ampulla of Vater; Pancreatoduodenectomy.

\title{
REFERÊNCIAS
}

1. Vallance S. Duodenectomy without pancreatectomy for extensive benign villous adenoma of the duodenum. Aust N Z J Surg. 1990; 60:311-314.

2. Sand JA, Nordback IH. Transduodenal excision of benign adenoma of the papilla of Vater. Eur J Surg 1995; 161:269-272.

3. Abolhoda AM, Yelcick JM, Swan KG. Pancreatoduodenectomy for giant benign villous adenoma of the ampulla of Vater. Am Surg 1997; 63:392-394.

4. Gibbs ER, Walton GF, Kent RB et al. Villous tumors of the ampulla of Vater Am Surg. 1997; 63:467-471.
5. Maher MM, Yeo CJ, Lillemore KD et al. Pancreas sparing duodenectomy for infra-ampullary duodenal pathology. Am J Surg 1996; 171:62-67.

Endereço para correspondência:

Dr. Guilherme Pinto Bravo Neto

Rua Severiano da Fonseca, 97

21931-220 - Rio de Janeiro-RJ 